\title{
Systolic Aortic Root Motion and Angle as novel indices of Left Ventricular Systolic Function
}

ghada m soltan ( $\square$ soltanghada@yahoo.com )

menofiya univerisity https://orcid.org/0000-0001-7649-0243

ahmed $m$ el kersh

menofiya univerisity

nevien e sami

faculity of medicine menofiua univerisity

ghadeer $\mathrm{m}$ yehia

shibin el kom teaching hospital

mahmoud ali soliman

Menoufia University Faculty of Medicine

Research

Keywords: Systolic anterior motion of aortic root, LV systolic function, strain, strain rate

Posted Date: January 8th, 2020

DOI: https://doi.org/10.21203/rs.2.20344/v1

License: (9) This work is licensed under a Creative Commons Attribution 4.0 International License.

Read Full License 


\section{Abstract}

\section{Background}

Aortic root motion was used only as a surrogate parameter of global left ventricular systolic function depending on its direct proportion to cardiac output. We hypothesize that aortic root motion angle and aortic root motion amplitude may overcome many limitations of EF calculation by $\mathrm{M}$ mode and two dimensional methods and are easier and reproducible.

\section{Objective}

The aim of this study is to asses systolic aortic root motion measured by $\mathrm{M}$ mode and aortic root motion angle as novel indices of global left ventricular systolic function.

Patients and methods

one hundred patients were enrolled in this study and divided into four groups: according to their age (above and below 60 years) and EF (above and below50\%). They were subjected to full history taking, careful clinical examination, and conventional echo-Doppler study.Systolic aortic root motion obtained from long axis parasternal view by M-mode echo guided by 2D echo, and aortic root motion angle was traced off line and mathematically measured. Also global logitudinal strain (GLS) and global longitudinal strain rate (GLSR) from apical 4,3 and 2 chamber views were measured offline.

Results

Statistical analysis of collected data show that there are significant differences between control groups and patient groups in aortic root motion angle ( $t=16.9$ and $p$ value $<0.001$, and in aortic root motion amplitude $(\mathrm{t}=20.1$ and $\mathrm{p}$ value $<0.001)$. Aortic root motion $(\mathrm{cm})$ and aortic root motion angle have significant positive correlation with $\mathrm{EF}(\mathrm{Mm}), \mathrm{EF}(2 \mathrm{D}), \mathrm{Fs}$, global longitudinal strain(GLS) and global Strain rate. The best cutoff value of aortic root motion angle was 19.5 degree, with sensitivity of $93.9 \%$, specificity of 96.1.Aortic root motion angle $>19.5$ predicts systolic function $>50 \%$ and that $<19.5$ predicts systolic function $<50 \%$ The best cutoff value of aortic root SAM was $8.5 \mathrm{~mm}$. An aortic root SAM of $\otimes 8.5$ mm predicts an LVEF of $₫ 50 \%$ with sensitivity of $95.9 \%$, specificity of $96.1 \%$.

\section{Conclusion}

The amplitude of systolic aortic root motion (SARM) by (M-mode) and aortic root motion angle are wellcorrelated with the EF and GLS and could be considered as novel indices of global left ventricular systolic function with high accuracy and reproducibility .

\section{Introduction}


Assessment of left ventricular (LV) systolic function is important for diagnosis, management, follow-up, and prognostic evaluation of patients in a variety of clinical settings. Accurate and reproducible determination of LV systolic function is of paramount importance in clinical practice.(1-2)

There are currently several echocardiographic measures of LV function available, as methodology has moved from linear measurements, via two-dimensional (2D) echocardiography with volume estimation and regional and global deformation analysis, to three-dimensional (3D) echocardiography(3-4). Assessment of LV function is challenging even with better and more automated methods. Ideally, one wants a sensitive and accurate measurement of LV function that correlates with the patient's symptoms, provides important information about the course of illness and can guide therapy. There is, however, no such perfect parameter (5-6).

The different components of myocardial fiber architecture contributing to a normal systolic function are unequally affected by different cardiac diseases, making different echocardiographic measures of LV systolic function more or less sensitive to detect subtle myocardial dysfunction. All the left ventricle function parameters measure contraction or volumes, and are therefore affected by loading conditions, which must be taken into account in every interpretation of LV function. (7-8).

The aortic root is the anatomical bridge between the left ventricle and the ascending aorta .The anterior and posterior walls of the aortic root are recognized as a pair of parall linear signals which move anteriorly in systole and posteriorly in diastole. (9-10). It is subjected to the same shearing force as left ventricle so we hypothesize that angle of its motion and also the amplitude of its motion are in direct proportion to systolic function of the left ventricle .

\section{Patients And Methods}

This prospective case-control study included (100) subjects; chosen according to inclusion and exclusion criteria. Subjects were recruited from cardiology department, Menoufia University Hospitals during the period from March 2018 to March 2019 after approval from the Research and Ethics Committee of Faculty of Medicine, Menoufia University. All participants included in the study signed informed written consent. Data were collected in a pre-organized data sheet (Case Sheet) by the researcher; from patients fulfilling the inclusion and exclusion criteria.

Patients and controls were classified into the following groups:

1. Group I: 25 patients above age of 60 years and ejection fraction above $50 \%$.

2. Group II: 25 patients below age of 60 years and ejection fraction above $50 \%$.

3. Group III: 25 patients below age of 60 years and ejection fraction below $50 \%$.

4. Group IV: 25 patients above age of 60 years and ejection fraction below $50 \%$.

The exclusion criteria were Patients with Aortic root calcification, dilated aortic root (more than $40 \mathrm{~mm}$ ), Aortic dissection, Aortic aneurysm, Aortic valve disease, Hypertrophic cardiomyopathy, Congenital 
anomalies of big vessels, and Post cardiothoracic surgery.

All participants were subjected to thorough history taking, clinical general and local examination, transthoracic conventional echocardiography using Vivid 9, General Electric Healthcare (GE Vingmed, Norway) equipped with a harmonic M5S variable-frequency $(1.7-4 \mathrm{MHz})$ phased-array transducer. All subjects were examined in supine and left lateral positions. Images were obtained from the left parasternal long axis view, apical 4-chamber view, apical 3 chamber, and apical 2 chamber view according to recommendations of American Society of Echocardiography and connected to single lead ECG (11). Strain and strain rate were also done, at least three cardiac cycles were digitally stored for offline analysis on a personal computer (PC) workstation using custom analysis software (Echopac PC, Version11.0X, GE Healthcare). Stored images were applied to automated Cardiac Motion Quantification (aCMQ) program.

The following measures were taken: (1) Left ventricular end-diastolic diameter (LVEDD), left ventricular end-systolic diameter (LVESD), (2) Left ventricular ejection fraction (LVEF) by M-mode in the parasternal long axis view and by 2D by modified Simpson method in apical 4 chamber and 2 chamber views. Patients with LVEF of $₫ 50 \%$ were considered to have impaired LV systolic function. (3) Fractional shortening (FS) by M-mode in the parasternal long axis view (4) Left atrial (LA) diameter and aortic root diameter, by M-mode in parasternal long axis views at the level of aorta-left atrium. (5) Mitral valve flow, and aortic valve flow assessment (6) strain and strain rate assessment (7) systolic Aortic root anterior motion amplitude by $\mathrm{m}$ mode in parasternal long axis views at the level of aorta-left atrium. (8) Aortic root motion angle is analyzed by printing the parasternal long axis views at the level of aorta-left atrium then was traced and was measured off side by the protractor. The latest two parameters can be easily added to built in calculation package in all echocardiographic machines (Fig 1)

\section{Statistical analysis}

Data were collected and coded then entered into a spread sheet using Microsoft Excel for windows office 2010. Data were statistically analyzed using the Statistical Package of Social Science (SPSS).

Quantitative data expressed as the mean \pm standard deviation (SD) or standard error (SE). SE=SD/square root of patients' number which was used in case of big SD, data were analyzed by independent sample, paired t-test and one-way analysis of variance (ANOVA) followed by Tukey test whenever single-step multiple comparisons were needed to maintain the integrity of data. While, qualitative data were expressed as number and percentage and were analyzed by Chi-square $\left(\mathrm{X}^{2}\right)$ test. The correlation was done using a Pearson correlation test. The receiver operating characteristic (ROC) curve and $95 \%$ confidence interval $(\mathrm{Cl})$ was performed to determine cutoff values for the studied measures. Sensitivity and specificity were determined. P-value was considered significant if $<0.05$ and highly significant if $<$ 0.001 .

\section{Results}




\section{Participants}

The study was conducted on one hundred subjects (51\%) of them were males and $(49 \%)$ of them were females with mean age of 68.6 years in group I, 35.6 years in group II, 48.2 years in group III, 67.2 years in group IV with a statistically-insignificant difference between the studied groups regarding age \& sex distribution

\section{Echocardiographic characteristics}

Mean Aortic root motion angle is 15 ,mean aortic root motion (m mode) is $0.67 \mathrm{~cm}$ mean global longitudinal strain is -11.3 .

Table (1): Quantitative descriptive statistics of measured echocardiographic parameters

\begin{tabular}{|lllll|}
\hline Parameter & Minimum & Maximum & Mean & SD \\
\hline LVEF by M-mode (\%) & 27 & 78 & 51.6 & 15.9 \\
\hline LVEF by 2D (\%) & 25 & 71 & 49.2 & 14.7 \\
\hline FS (\%) & 15 & 54 & 32.2 & 10.8 \\
\hline Aortic root diameter (mm) & 3,1 & 3,2 & 3.33 & 0.57 \\
\hline LA diameter (mm) & 3.2 & 4.7 & 2.9 & 2.2 \\
\hline Aortic root motion angle & 3 & 30 & 15 & 5.27 \\
\hline $\begin{array}{l}\text { Aortic root motion } \\
\text { m mode }\end{array}$ & 0.3 & 1.6 & 0.67 & 0.28 \\
\hline Global longitudinal strain & -6.3 & -24.5 & -11.3 & 3.77 \\
\hline
\end{tabular}

\section{Regarding aortic root motion angle and amplitude}

Significant differences between groups $1 \& 2$ and groups $3 \& 4$ in aortic root motion angle with $t=16.945$ and $p$ value $<0.001$, Significant differences between groups $1 \& 2$ and groups $3 \& 4$ in aortic root motion amplitude with $t=20.1$ and $p$ value $<0.001$.

Also there are Significant differences between groups $1 \& 2$ and patient groups $3 \& 4$ in global longitudinal Strain with $t=-13.19$ and $p$ value $<0.001$ 
Table (2):T-Test of Global longitudinal Strain, Aortic Root Motion m mode and Aortic Root Motion angle in control groups $(1,2)$ and patient groups $(3,4)$.

\begin{tabular}{|llllll|}
\hline \multicolumn{2}{|l}{ parameter } & Mean & $N$ & $\begin{array}{l}\text { Std. } \\
\text { Deviation }\end{array}$ & $\begin{array}{l}\text { Std. Error } \\
\text { Mean }\end{array}$ \\
\hline $\begin{array}{l}\text { Pair } \\
1\end{array}$ & Global longitudinal Strain G1and2 & -18.09 & 50 & 2.82 & 0.489 \\
\cline { 2 - 6 } Gair & Global longitudinal Strain G3and4 & -10.98 & 50 & 3.78 & 0.546 \\
2 & Aortic Root Motion m mode (cm) G1and2 & 1.23 & 50 & 0.22 & 0.031 \\
\cline { 2 - 6 } Pair & Aortic Root Motion m mode (cm) G3and4 & 0.56 & 50 & 0.119 & 0.016 \\
3 & $\begin{array}{l}\text { Aortic Root Motion angle } \\
\text { (degree)G1and2 }\end{array}$ & 20 & 50 & 3.27 & 0.462 \\
$\begin{array}{l}\text { Aortic Root Motion angle (degree } \\
\text { G3and4 }\end{array}$ & 8 & 50 & 4.07 & 0.576 \\
\hline
\end{tabular}

Table (3): Paired Samples Differences Test regarding global longitudinal strain , aortic root motion (mm) and aortic root motion angle.

\begin{tabular}{|c|c|c|c|c|c|c|c|c|}
\hline \multicolumn{2}{|c|}{ Parameter } & \multicolumn{5}{|c|}{ Paired Differences } & \multirow[t]{3}{*}{$t$} & \multirow{3}{*}{$\begin{array}{l}\text { P- } \\
\text { value }\end{array}$} \\
\hline \multirow[t]{5}{*}{ Mean } & \multirow[t]{2}{*}{ SD } & \multirow[t]{2}{*}{$\begin{array}{l}\text { Std. } \\
\text { Error } \\
\text { Mean }\end{array}$} & \multicolumn{2}{|c|}{$\begin{array}{l}95 \% \text { Confidence } \\
\text { Interval of the } \\
\text { Difference }\end{array}$} & \multirow[b]{3}{*}{-8.19} & \multirow[b]{3}{*}{-6.02} & & \\
\hline & & & Lower & Upper & & & & \\
\hline & $\begin{array}{l}\text { Global } \\
\text { longitudinal } \\
\text { Strain }\end{array}$ & -11.3 & 3.77 & 0.538 & & & -13.19 & .000 \\
\hline & $\begin{array}{l}\text { Aortic root motion } \\
\mathrm{m} \text { mode }(\mathrm{cm})\end{array}$ & 0.67 & 0.281 & 0.039 & 0.594 & 0.75 & 16.94 & .000 \\
\hline & $\begin{array}{l}\text { Aortic Root } \\
\text { motion } \\
\text { angle(degree) }\end{array}$ & 15 & 5.27 & 0.745 & 13.50 & 16.49 & 20.11 & .000 \\
\hline
\end{tabular}

Table (4): Descriptive Statistics of Aortic Root motion angle in different groups. 


\begin{tabular}{|lllllll|}
\hline Parameter & N & Minimum & Maximum & Mean & Std. Deviation & P Value \\
\hline Aortic Root motion angle G1 & 25 & 18 & 30 & 22.88 & 3.25 & 0.00 \\
\hline Aortic Root motion angle G2 & 25 & 20 & 30 & 23.56 & 2.87 & 0.00 \\
\hline Aortic Root motion angle G3 & 25 & 5 & 18 & 9.92 & 4.31 & 0.00 \\
\hline Aortic Root motion angle G4 & 25 & 3 & 15 & 6.52 & 2.93 & 0.00 \\
\hline
\end{tabular}

Aortic root motion angle has a Statistically significant higher value in healthy group(1 and 2)than patient in group (3 and 4 )

Table (5): Descriptive Statistics of Aortic Root Motion m mode (cm) in different groups .

\begin{tabular}{|c|c|c|c|c|c|c|}
\hline Parameter & $\mathbf{N}$ & Minimum & Maximum & Mean & $\begin{array}{l}\text { Std. } \\
\text { Deviation }\end{array}$ & $\begin{array}{l}P \\
\text { value }\end{array}$ \\
\hline $\begin{array}{l}\text { Aortic Root Motion m mode } \\
(\mathrm{cm}) \mathrm{G} 1\end{array}$ & 25 & 0.9 & 1.60 & 1.19 & 0.228 & 0.0001 \\
\hline $\begin{array}{l}\text { Aortic Root Motion m } \\
\text { mode }(\mathrm{cm}) \mathrm{G} 2\end{array}$ & 25 & 0.8 & 1.60 & 1.28 & 0.211 & 0.00 \\
\hline $\begin{array}{l}\text { Aortic Root Motion m } \\
\text { mode }(\mathrm{cm}) \mathrm{G} 3\end{array}$ & 25 & 0.4 & 0.8 & 0.58 & 0.117 & 0.00 \\
\hline $\begin{array}{l}\text { Aortic Root Motion m mode } \\
\text { (cm) G4 }\end{array}$ & 25 & 0.4 & 0.9 & 0.54 & 0.119 & 0.00 \\
\hline
\end{tabular}

Aortic Root Motion angle has a significant positive correlation with EF_Mm, EF-2D, Fs, global longitudinal strain (GLS) and global Strain rate .and the same regarding Aortic root motion height (m mode).

Table (6) correlations between Aortic Root Motion ( M- mode) and Aortic Root motion angle and other echocardiographic parameters . 


\begin{tabular}{|llll|}
\hline Parameter & RootMotion_M-mode_cm & Aortic_Root_motion_angle \\
\hline EF_Mm & $\mathrm{R}$ & 0.77 & 0.83 \\
\cline { 2 - 4 } & P value & 0.000 & 0.00 \\
EF_2D & $\mathrm{R}$ & 0.79 & 0.85 \\
& $\mathrm{P}$ value & 0.000 & 0.00 \\
Fs & $\mathrm{R}$ & 0.78 & 0.69 \\
& P value & 0.001 & 0.001 \\
GLS & $\mathrm{R}$ & 0.85 & 0.82 \\
& P value & 0.000 & 0.000 \\
GLSR & $\mathrm{R}$ & 0.79 & 0.73 \\
& P value & 0.004 & 0.005 \\
\hline
\end{tabular}

Table (7): Sensitivity, Specificity and cutoff value of Global long longitudinal Strain, aortic root motion $(\mathrm{mm})$ and aortic root motion angle

\begin{tabular}{|llll|}
\hline & $\begin{array}{l}\text { Global long longitudinal } \\
\text { Strain }\end{array}$ & $\begin{array}{l}\text { aortic root motion m mode } \\
(\mathbf{c m})\end{array}$ & $\begin{array}{l}\text { Aortic Root Motion } \\
\text { Angle(degree) }\end{array}$ \\
\hline Sensitivity & $89.8 \%$ & $95.9 \%$ & $93.9 \%$ \\
\hline Specificity & $88 \%$ & $96.1 \%$ & $96.1 \%$ \\
\hline CUTOFF & -13.25 & 0.85 & 19.5 \\
\hline
\end{tabular}

This Table demonstrate the best cut off point value of Global Longitudinal Strain >-13.52. The sensitivity and specificity were $89.8 \%$ and $88 \%$ respectively. Moreover, the best cut off point value of aortic root motion (m mode) $>0.85 \mathrm{~cm}$. The sensitivity and specificity were $95.9 \%$ and $96.1 \%$ respectively. Moreover, the best cut off point value of Aortic Root Motion Angle > 19.5 degree with sensitivity and specificity are $93.9 \%$ and $96.1 \%$ respectively.

\section{Discussion}

In this study we tried to use systolic aortic root motion ( $\mathrm{M}$ mode)and aortic root motion angle as novel indices of global left ventricular systolic function In the 100 patients included in this study ,there are Significant differences between control groups and patient groups in aortic root motion ( $\mathrm{m}$ mode ) with $\mathrm{t}$ $=20.1$ and $p$ value $<0.001$ and Significant differences between two groups in aortic root motion angle with $t=16.945$ and $p$ value $<0.001$. Also there are Significant differences between control groups and patient groups in global long longitudinal Strain with $t=-13.19$ and $p$ value $<0.001$. 
Aortic root motion m mode $(\mathrm{cm})$ and aortic root motion angle have significant positive correlation with $\mathrm{EF}(\mathrm{Mm}), \mathrm{EF}(2 \mathrm{D}), \mathrm{Fs}$, global longitudinal strain(GLS) and global longitudinal Strain rate .

The best cutoff value of aortic root SAM was $8.5 \mathrm{~mm}$. An aortic root SAM of $\otimes 8.5 \mathrm{~mm}$ predicts an LVEF

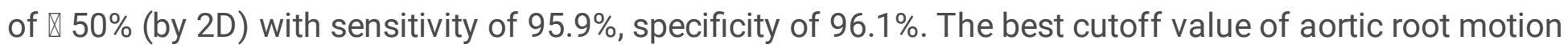
angle was $19.5 \mathrm{~mm}$, with sensitivity of $93.9 \%$, specificity of $96.1 \%$.

Regarding correlation between EF and global longitudinal strain, Our finding agrees with study done in 2018 by Luigi et al. He retrospectively identified 96 patients with a reduced LVEF $<50 \%$ (screening echocardiogram), whose LVEF had increased by at least $10 \%$ and normalized $(>50 \%)$ on evidence based medical therapies (baseline echocardiogram). He examined absolute global longitudinal strain (GLS) on the baseline echocardiogram in relation to changes in LVEF on a follow-up echocardiogram; Patients with recovered LVEF had a wide range of GLS. The GLS on the baseline study correlated with the LVEF at the time of follow-up ( $r=0.33, p<0.001)$. The likelihood of having an LVEF $>50 \%$ on follow-up increased by $24 \%$ for each point increase in absolute GLS on the baseline study (or 1.24, $p=0.001$ ).An abnormal GLS $(\leq 16 \%)$ at baseline had a sensitivity of $88 \%$, a specificity of $46 \%$, and an accuracy of $0.67(p<0.001)$ as a predictor of a decrease in LVEF $>5 \%$ during follow up. A normal GLS $(>16 \%)$ on the baseline study had a sensitivity of $47 \%$, a specificity of $83 \%$ and an accuracy of $0.65(p=0.002)$ for predicting a stable LVEF (- 5 to $5 \%)$ on follow-up.

He concluded that; in patients with a recovered LVEF, an abnormal GLS predicts the likelihood of having a decrease LVEF during follow-up, whereas a normal GLS predicts the likelihood of stable LVEF during recovery.

Recordings of aortic root movement represent one of the first accomplishments of ultrasound in medicine and mark the beginning of functional cardiac imaging. However, the underlying mechanism is not completely understood (13). Since the aortic root is directly connected to the cardiac skeleton Matthias A et al, 2019 hypothesized, that the amplitude of systolic aortic root motion (SARM) may be mainly caused by displacement of the cardiac base towards the apex and might therefore be used as measure of left ventricular longitudinal function (LV-LF). There study was done on 180 patients with dilated cardiomyopathy and 180 healthy controls were prospectively included into this study. They founded that SARM was lower in patients compared to controls $(9 \pm 3 \mathrm{~mm}$ vs. $12 \pm 2 \mathrm{~mm}, \mathrm{p}<0.001)$ and lowest in patients with cardiovascular events $(9 \pm 3 \mathrm{~mm}$ vs. $7 \pm 3 \mathrm{~mm}, p<0.001)$. During a median follow-up time of 38 months, the combined end-point of cardiovascular death or hospitalization for heart failure was reached by 25 patients (13.9\%). Reduced SARM had significant prognostic impact on outcome (hazard ratio $0.74,95 \%$ confidence interval $0.63-0.88, p<0.001$ ) and remained an independent predictor in the multivariate analysis. Compared to parameters with potential influence on its mechanism, SARM correlated best $(r=0.75, p<0.001)$ with global longitudinal strain (GLS). SARM may therefore represent an alternative echocardiographic parameter for the assessment of LV-LF, particularly when GLs is not feasible or apical views are not available. Due to its high echogenicy, SARM can easily be visualized by M-mode echocardiography. Matthias A et al, 2019 demonstrated that SARM is not exclusively directed 
anterior but shows an additional downward and lateral-left displacement similar to the systolic movement of the cardiac base towards the apex, Abnormal SARM is a frequent finding in cardiac disease. Alterations are, however, not specific to a particular pathology but can generally be regarded as a prognostically unfavorable sign in patients with systolic heart failure, Matthias A et al, 2019 data suggest that SARM is closely related to global longitudinal strain and thus might represent an alternative measure of LV longitudinal function especially when strain assessment is not feasible or available.

In our study we founded that SARM was lower in patients compared to controls $(5.8 \pm 1.17 \mathrm{~mm}, 5.44 \pm$ 1.19 in patients group 3 and 4 vs. $11.9 \pm 2.2,12.8 \pm 2.1 \mathrm{~mm}, p<0.001$ in controls group). Our findings agree with Matthias A et al, 2019 and demonstrates the difference between patient Groups in Values of Root Motion $\mathrm{m}$ mode height which specify a Statistically significant higher value in healthy group (1 and 2 ) than patient in group ( 3 and 4$)$ where EF $2 d$ less than $50 \%$.SARM correlated best $(r=0.814, p<0.001)$ with global longitudinal strain (GLS) which also agrees with Matthias A et al, 2019.

Another study done by Ahmadou M et al, 2018 on correlations of left ventricular systolic function indices with aortic root systolic excursion (ARSE) in which Overall 213 echocardiograms were included from 106 males (49.8\%), with mean age of the participants being 52.4 (SD: 18.3) years. The rate of LV systolic dysfunction was highest with Teicholz method (17.4\%) and lowest with MAPSE method (5.2\%). ARSE correlated with the LV functions. This was highest for the Simpson method $(r=0.619, p<0.001)$, and lowest for the ITV method $(r=0.319, p<0.001)$. Optimal cut-offs to detect LV systolic dysfunction was $\approx$ $6.6 \mathrm{~mm}$. For an LV ejection fraction $<55 \%$, the sensitivity was $82.9 \%$, and the specificity was $97.2 \%$, demonstrated that ARSE correlated well with LV systolic function which agrees with our finding as we founded that aortic root motion $m$ mode diameter has a significant correlation with EF_Mm, EF-2D, Fs, SV, Global longitudinal strain and strain rate.

Our findings agree also with Osama A Diab,2015 who study the quantitative assessment of Aortic Root Systolic Anterior Motion as an echocardiographic measure of global left ventricular systolic function.

The study included 38 males and 19 females with mean age of $49.8 \pm 16.4$ years. Mean aortic root SAM was $9.3 \pm 2.8 \mathrm{~mm}$ (range: $2-15 \mathrm{~mm}$ ). Mean M-mode derived LVEF was $49.3 \pm 17.9 \%$, and 2D derived LVEF was $46.2 \pm 16.9 \%$. Mean FS was $28.2 \pm 12.5 \%$. Mean Aortic cusp separation (ACS) was $16.8 \pm 3.3 \mathrm{~mm}$. In 15 patients $(26.3 \%)$ there was SWMA, while the remaining 42 patients $(73.7 \%)$ had normal segmental wall motions. Aortic root SAM was strongly correlated with LVEF by M-mode $(r=0.77, p=0.00)$, LVEF by $2 D(r=0.78, p=0.00)$, FS $(r=0.79, p=0.00)$, and ACS $(r=0.56, p=0.00)$. These correlations were better in patients with normal wall motions than those with SWMA. Diab O.A. 2015 found that the best cutoff value of aortic root SAM was $9.5 \mathrm{~mm}$. An aortic root SAM of $\triangle 9.5 \mathrm{~mm}$ predicts an LVEF of $\triangle 50 \%$ (by 2D) with sensitivity of $92 \%$, specificity of $96.7 \%$, and total validity of $94.5 \%$.

To the best of our knowledge It is the first time to study Aortic Root Motion angle correlation to left ventricular systolic function indices and we founded that Aortic Root Motion angle has a significant correlation with EF_(M mode, EF-2D), Fs and global (GLS) Strain and strain rate. 
We founded that Aortic Root Motion angle was lower in patients compared to controls $(9.9 \pm 4.4,6.5 \pm$ $2.9)$ in patients groups 3 and 4 vs. $(22.9 \pm 3.6,23.6 \pm 2.9)$ in control groups 1 and 2$)$.

Study of ROC curve of Root Motion m mode diameter, Aortic Root Motion angle and global (LSGG) Strain yield that the Cut-off value of Root Motion m mode diameter was $8.5 \mathrm{~mm}$ with specificity $96.1 \%$ and sensitivity $95.9 \%$, the Cut-off value of Aortic Root Motion angle was $19.5 \mathrm{~mm}$ with specificity $96.1 \%$ and sensitivity $93.9 \%$ and the Cut-off value of global (LSGG) Strain was $13.25 \mathrm{~mm}$ with specificity $88 \%$ and sensitivity $89.8 \%$.

\section{Limitations}

This technique cannot be generalized to all patients as we excluded patients with aortic calcifications which are common finding in old age patients. Also patients with aortic valve disease however many cases of aortic valve affection is due to rheumatic pathology especially in low-income patients .

\section{Conclusion}

The amplitude of systolic aortic root motion (SARM) by (M-mode) and aortic root motion angle are wellcorrelated with the EF and GLS and could be considered as novel indices of global left ventricular systolic function with high accuracy and reproducibility

\section{List Of Abbreviations}

1-EF\% ejection fraction

2-G LS global longitudinal strain

3-GLSR global longitudinal strain rate

4- $\mathrm{Mm} \quad \mathrm{M}$ - mode

5- FS fractional shortening

6- (SARM) systolic aortic root motion

(LV-LF) left ventricular longitudinal function

7-LVEF left ventricular ejection fraction

8- LV left ventricle

9-2D two- dimensional

10-3D three- dimensional 
11-LVESD left ventricular end systolic dimension

12-LVEDD left ventricular end diastolic dimension

13- (a CMQ) automated Cardiac Motion Quantification

14-(ARSE) aortic root systolic excursion

15-MAPSE Mitral annulus plain systolic excertion

16- SWMA systolic wall motion abnormalities

17-(ACS) Aortic cusp separation

\section{Declarations}

Ethics approval and consent to participate: each patient participating in this study have written consent

Consent for publication: not appropriate for this research

Availability of data and material: all data related to these study will be available when there is appropriate indication

Competing interests: no competing interest

Funding: No fund

Authors' contributions: corresponding author, collect data , patient recruitment, clinical and echocardiography study

Acknowledgements: Non.

No conflict of interest

\section{References}

1. Ponikowski P, Voors AA, Anker SD, Bueno H, Cleland JGF, Coats AJS, Falk V, González-Juanatey JR, Harjola VP, Jankowska EA, Jessup M, Linde C, Nihoyannopoulos P, Parissis JT, Pieske B, Riley JP, Rosano GMC, Ruilope LM, Ruschitzka F, Rutten FH, van der Meer P; ESC Scientific Document Group (2016) The Task Force for the diagnosis and treatment of acute and chronic heart failure of the 
European Society of Cardiology (ESC) Developed with the special contribution of the Heart Failure Association (HFA) of the ESC. Eur Heart J 37:2129-2200.

2. Yancy CW, Jessup M, Bozkurt B, Butler J, Casey DE Jr, Drazner MH, Fonarow GC, Geraci SA, Horwich T, Januzzi JL, Johnson MR, Kasper EK, Levy WC, Masoudi FA, McBride PE, McMurray JJ, Mitchell JE, Peterson PN, Riegel B, Sam F, Stevenson LW, Tang WH, Tsai EJ, Wilkoff BL (2013) 2013 ACCF/AHA guideline for the management of heart failure: executive summary: a report of the American College of Cardiology Foundation/American Heart Association Task Force on practice guidelines. Circulation 128:1810-1852.

3. Klaeboe LG , Edvardsen T. (2019) Echocardiographic assessment of left ventricular systolic function, $\mathrm{J}$ of Echocardiography 17:10-16.

4. Rastogi A, Novak E, Platts AE, Mann DL (2017) Epidemiology, pathophysiology and clinical outcomes for heart failure patients with a mid-range ejection fraction. Eur J Heart Fail 19:1597-1605.

5. Clarke CL, Grunwald GK, Allen LA, Barón AE, Peterson PN, Brand DW, Magid DJ, Masoudi FA (2013) Natural history of left ventricular ejection fraction in patients with heart failure. Circ Cardiovasc Qual Outcomes 6:680-686.

6. Dunlay SM, Roger VL, Weston SA, Jiang R, Redfield MM (2012) Longitudinal changes in ejection fraction in heart failure patients with preserved and reduced ejection fraction. Circ Heart Fail 5:720726.

7. Stokke TM, Hasselberg NE, Smedsrud MK, Sarvari SI, Haugaa KH, Smiseth OA, Edvardsen T, Remme EW Geometry as a confounder when assessing ventricular systolic function: comparison between ejection fraction and strain. J Am Coll Cardiol. 2017;70:942-54.

8. Thavendiranathan P, Poulin F, Lim KD, Plana JC, Woo A, Marwick TH (2014) Use of myocardial strain imaging by echocardiography for the early detection of cardiotoxicity in patients during and after cancer chemotherapy: a systematic review. J Am Coll Cardiol; 63:2751-2768.

9. Anderson RH (2006) Demystifying the anatomic arrangement of the aortic valve. Eur J Cardiothorac Surg 29: 1006- 1007.

10. Hagendorff A, Stoebe S, Tayal B (2018) A systematic approach to 3D echocardiographic assssment of the aortic root, Glob Cardiol Sci Pract. (2):12

11. Lang RM, Badano LP, Mor-Avi V, Afilalo J, Armstrong A, Ernande L, Flachskampf FA, Foster E, Goldstein SA, Kuznetsova T, Lancellotti P, Muraru D, Picard MH, Rietzschel ER, Rudski L, Spencer KT, Tsang W, Voigt JU (2015) Recommendations for cardiac chamber quantification by echocardiography in adults: An update from the American Society of Echocardiography and the European Association of Cardiovascular Imaging. J Am Soc Echocardiogr 28(1):1-39.e14.

12. Luigi Adamo, Andrew Perry, Eric Novak, Majesh Makan, Brian R. Lindman, and Douglas L. Mann , Abnormal Global Longitudinal Strain Predicts Future Deterioration of Left Ventricular Function in Heart Failure Patients With a Recovered Left Ventricular Ejection Fraction, Circulation Heart Failure June $2017,10(6)$ :e003788. 
13. Aurich $M$, Niemers $M$, Fuchs P, Greiner S, Müller-Hennessen M, Uhlmann L, Giannitsis E, Ehlermann P, Meder B2, Katus HA, Mereles D(2019) Pathophysiological background and prognostic implication of systolic aortic root motion in non-ischemic dilated Sci Rep 9 (1): 3866

14. Jingi AM, Hamadou B, Noubiap JJ, Mfeukeu-Kuate L, Boombhi J, Nganou CN, Ateba NA, Ndoadoumgue AL, Nyaga UF, Menanga A, Kingue S (2018) Correlations of left ventricular systolic function indices with aortic root systolic excursion (ARSE) : A cross-sectional echocardiographic study. PLoS One 13(11):e0206199

15. Ahdamo L, Perry A, Novak E, Makan M, Lindman BR, Mann DL (2017) Abnormal Global Longitudinal Strain Predicts Future Deterioration of Left Ventricular Function in Heart Failure Patients With a Recovered Left Ventricular Ejection Fraction. Circ Heart Fail 10(6):e003788 •

16. Diab OA (2015) Quantitative Assessment of Aortic Root Systolic Anterior Motion as an Echocardiographic Measure of Global Left Ventricular Systolic Function. Ain Shams University doi: 10.13140/RG.2.1.1966.6081

\section{Figures}

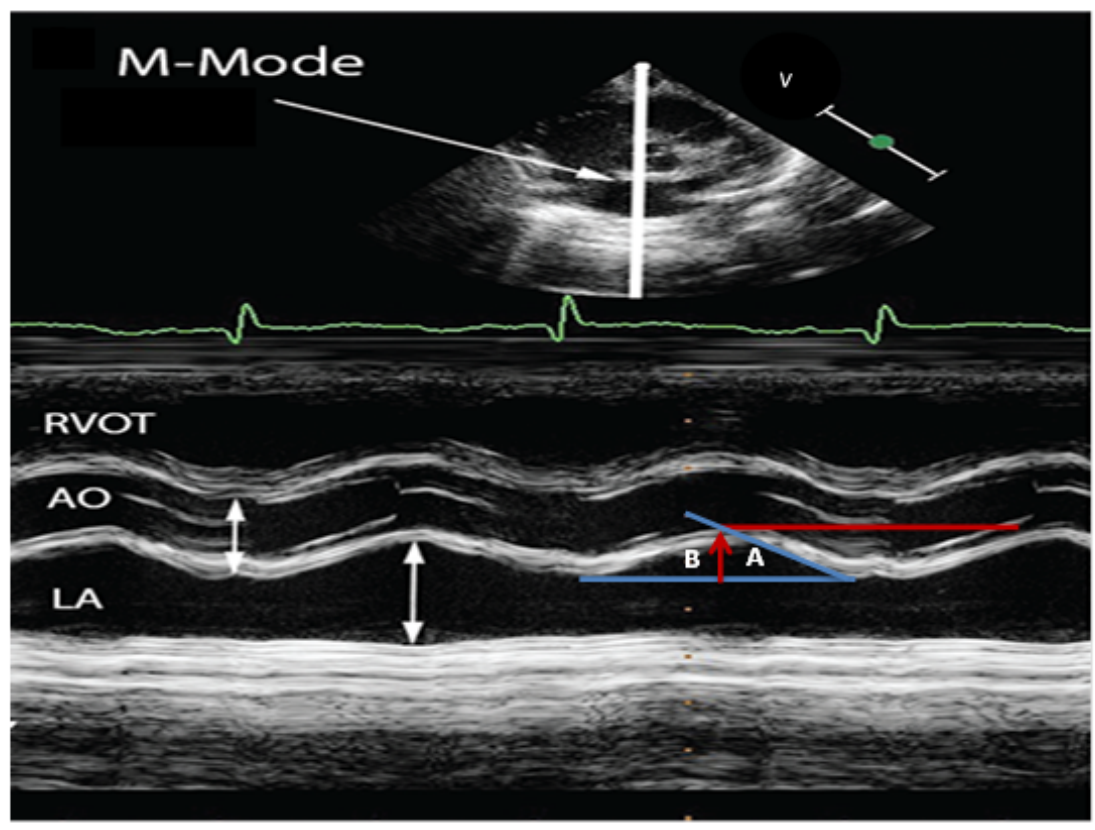




\section{Figure 1}

Illustration of Aortic root motion angle and aortic root motion height in patient with EF more than $50 \%$. (A) Aortic root motion angle is represented by the angle between two blue lines. (B) root motion height is represented by the red vertical arrow.

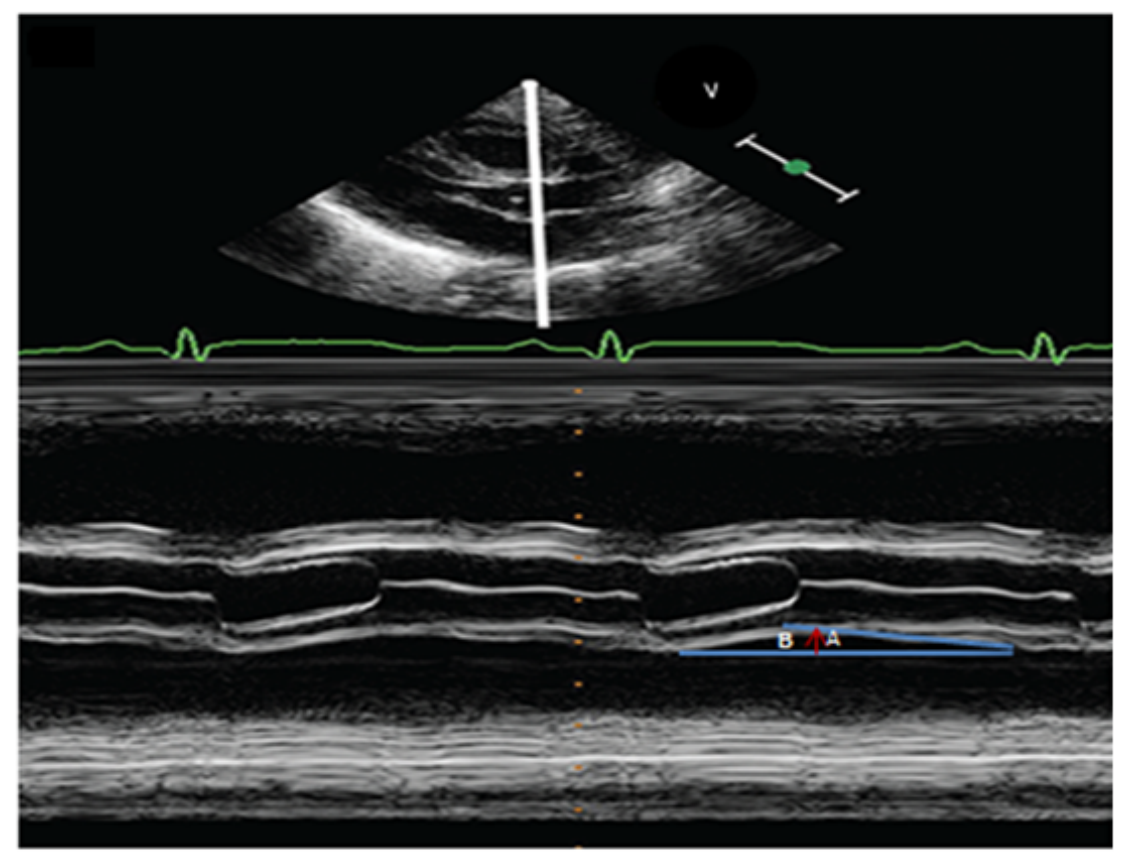

\section{Figure 2}

Illustration of Aortic root motion angle and aortic root motion height in patient with EF below $50 \%$. 


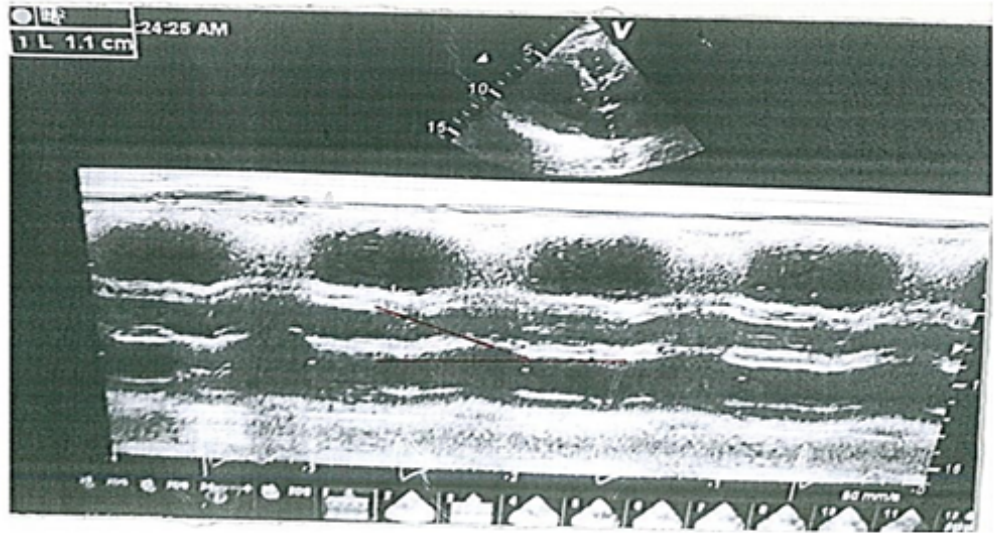

Figure 3

aortic root motion angle equals 25 degree and SARM equals $1.1 \mathrm{~cm}$ in patient in group(1) 


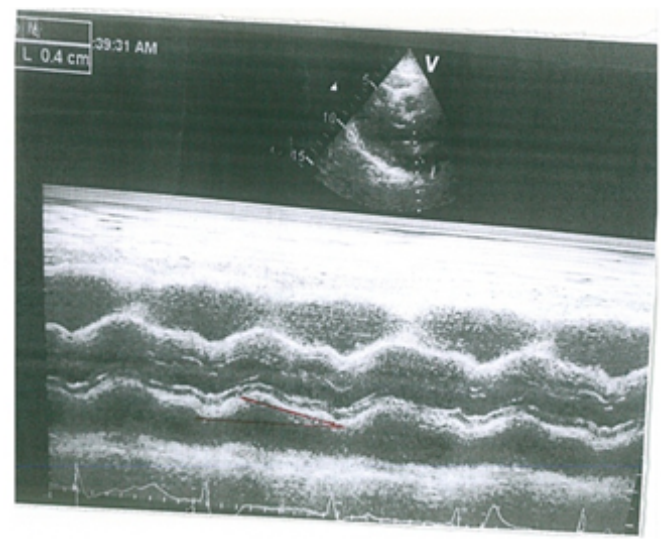

Figure 4

aortic root motion angle equals 10 degree and SARM equals $0.4 \mathrm{~cm}$ in patient in group( 4) 

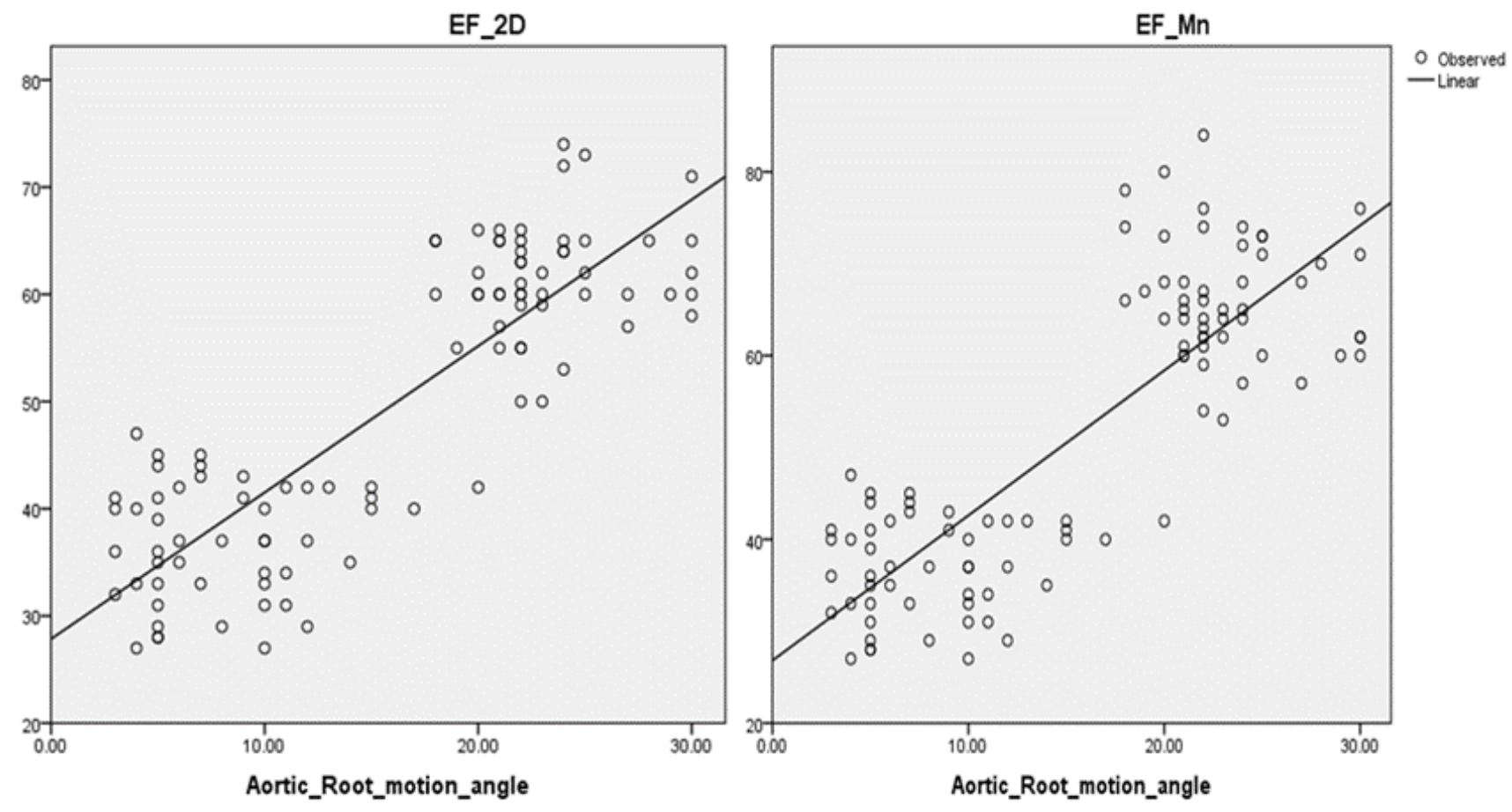

Figure 5

correlation of aortic root motion angle with $\mathrm{EF}$ (m.mode) and EF(2D 


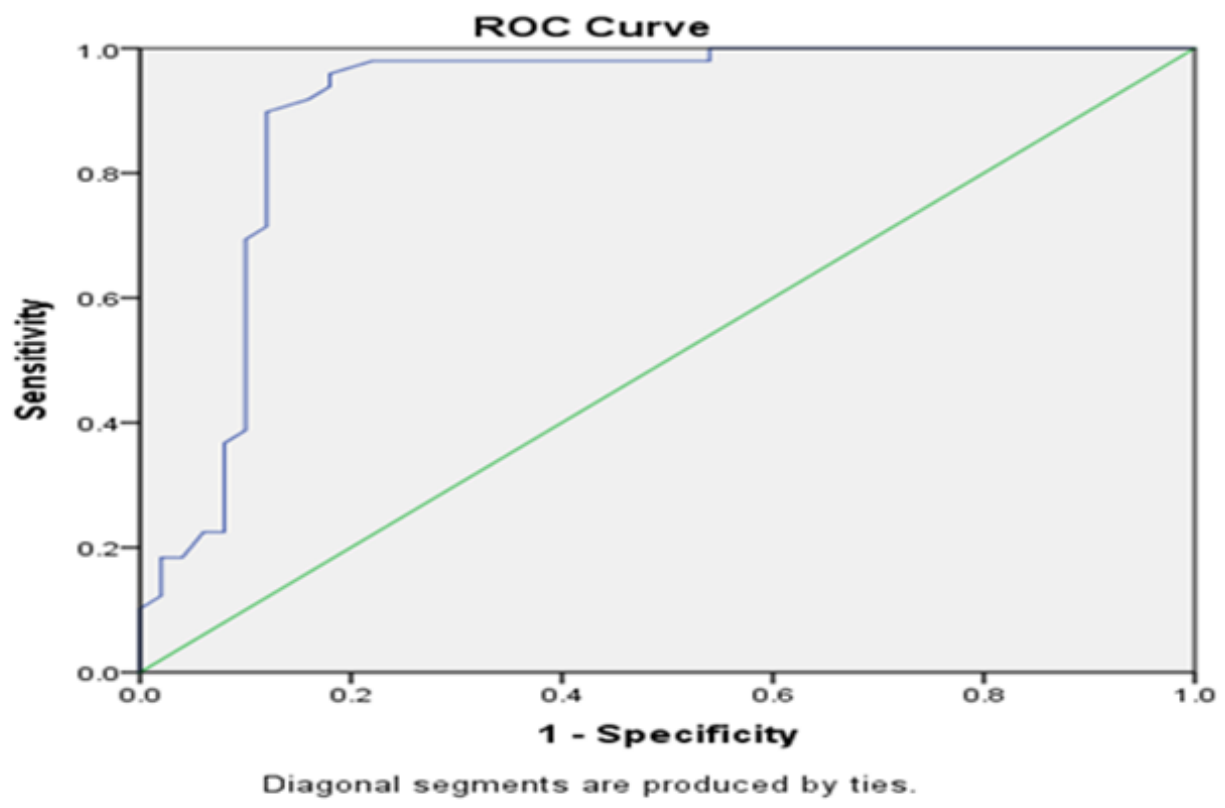

Figure 6

ROC curve for the best sensitivity and specificity of global longitudinal strain 


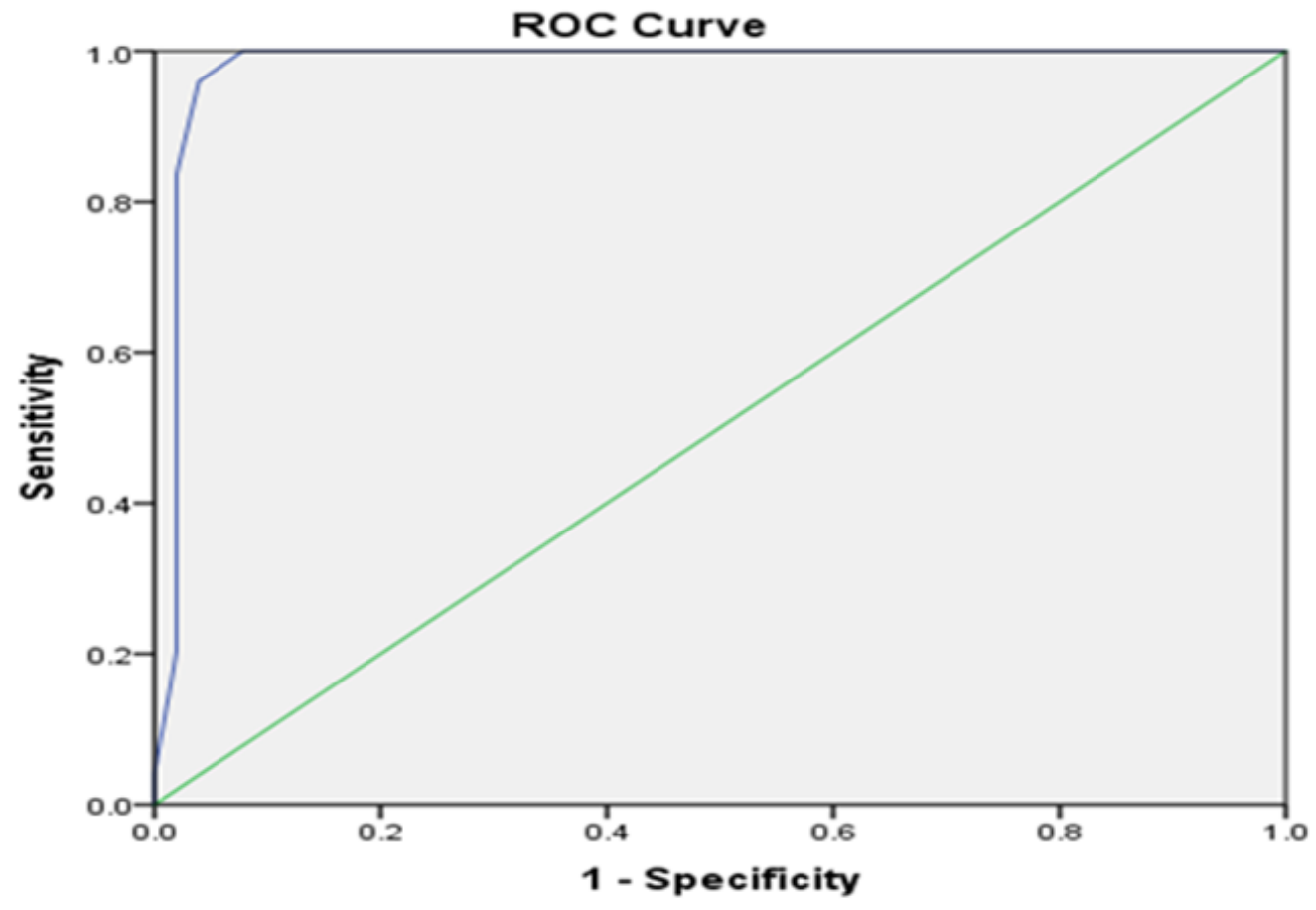

Diagonal segments are produced by ties.

Figure 7

ROC curve for the best sensitivity and specificity of Root Motion $\mathrm{m}$ mode $\mathrm{cm}$ 


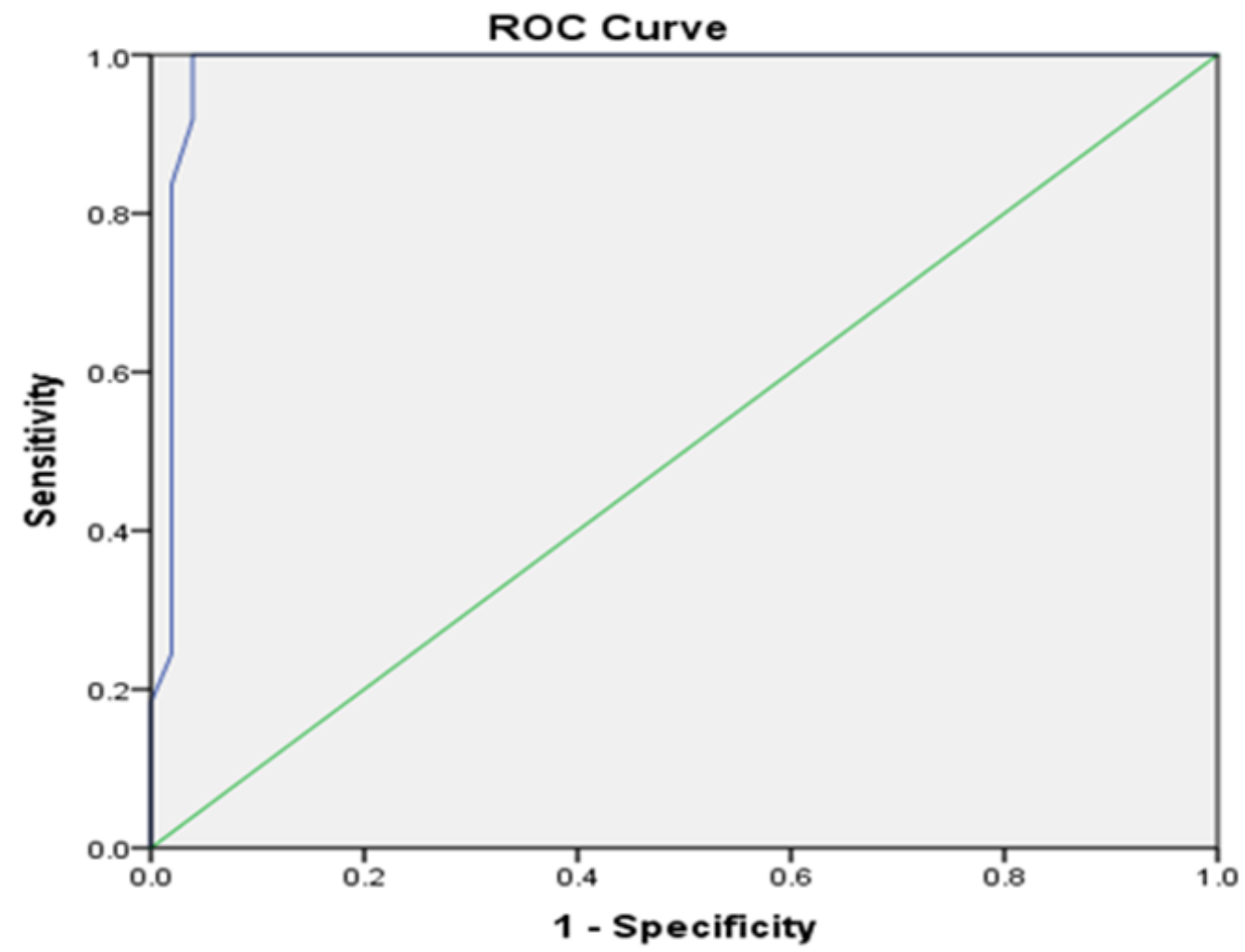

Diagonal segments are produced by ties.

\section{Figure 8}

ROC curve for the best sensitivity and specificity of aortic root motion angle. 